\title{
Decreasing Grey Matter in Multiple Sclerosis: A Serious Disorder in the Brain
}

Received: January 20, 2017; Accepted: February 19, 2018; Published: February 25, 2018

\section{Introduction}

Multiple sclerosis is one of the world's most common neurologic disorders. It can trouble the central nervous system that the brain the spinal cord and the optic nerve. It takes place when our own immune system attacks the myelin sheath which is the protective sheath around neurons. As a result, it contributes to the interruption of signals to the brain from the body. This procreate many neural, mental and movement disabilities. In many countries it is the leading cause of non-traumatic neurologic disability in young adults MS was previously considered to primarily affect the white matter (WM) [1]. But the recent studies prove the decreasing grey matter volume in neurodegenerative diseases like MS. Grey matter atrophy occurs in all MS phenotypes. Many researchers investigated the spatiotemporal pattern of GM with faster disability accumulation in MS.

\section{Recent Findings}

Former times the way of treatments for MS are quite limited. But now the research has framed an impulse and potential treatments and innovations are emerging always. Many Disease Modifying Therapies (DMT) are available to slow the course of MS. Some of the Recent studies also have implied that a number of factors, like iron accumulation, may be involved in MS plaque formation and damaging grey matter cells. The findings of the 2013 Atlas of MS survey, along with those of other recent surveys such as the European Multiple Sclerosis Platform MS Barometer, highlight the wide variation in access to treatment and service provision for people with MS both across the globe and within regions. In 2017 EDI approved a new pioneered drug named Ocrelizumab (Ocrevus) for the treatment of relapsing MS. Some of the recent researches suggest the use of cognitive training in reducing the cognitive symptoms of MS. Studies also prove the role of physical training in lessening the excessive fatigue and mobility impairments. The Oregon Health \& Science University in Portland put forward an antioxidant namely Lipoic acid which may prove valuable in the treatment of SPMS. An exciting remedy which is under research for the treatment of MS is the gut microbes.

\section{Discussion}

Deep grey matter and increased disability

The method of approach to scrutinize the tie between decrease

\begin{abstract}
Jes Paul*
Department of Molecular and Cellular Physiology, Albany Medical College, Albany, NY, USA
\end{abstract}

*Corresponding author: Jes Paul

” paulj5@amc.edu; jesrejoice@gmail.com

Department of Molecular and Cellular Physiology, Albany Medical College, Albany, NY, USA.

Tel: 4049802103

Citation: Paul J (2018) Decreasing Grey Matter in Multiple Sclerosis: A Serious Disorder in the Brain. J Transl Neurosci Vol.3 No.1:2

in the deep grey matter volume and increased disability in the MS victims was done by analysing the DGM from temporal, occipital, cerebral GM, brainstem, cerebral white matter of various phenotypes of MS patients. When studied along a large multi-central and longitudinal way, Disability accumulation in MS by DGM volume loss was remarked.

The clinical course of MS is heterogeneous. The different phenotypes of MS include (relapsing-remitting [RR] MS), where the patient experiences relapses with recovery, while others develop progressive disability either from the onset (primaryprogressive [PP] MS) or after a period of relapses (secondaryprogressive [SP] MS). Almost $90 \%$ of the cases are RRMS which later progress to SPMS. The Actual disability over time is determined by neurodegeneration, which plays a crucial role in MS.

In vivo Neurodegeneration results in decreased brain volume also known as brain atrophy which is measured by MRI. Over time, brain volume declines more rapidly in MS patient. More brain atrophies particularly seen in SPMS which is estimated to be $0.6 \%$ [2]. While monitoring response to treatments the role of brain atrophy in MS has been recently used a s a primary outcome measure in Phase II clinical trials in SPMS [3,4].

Whole brain atrophy is driven by the neuroaxonal loss in the GM [5]. Increased volume losses of GM contribute to long term disability. Cingulated cortex and thalamus are the two regions 
of GM which are mostly affected in MS [6,7]. Upsurge in the volume loss results in gained disability, which in turn results in escalation of cognitive impairment. Regional predilection for atrophy is not unique to MS. Some GM regions such as cortical lobes and temporal lobes show faster atrophy than others for example insula, hippocampus, thalamus, supplemental motor area, anterior and posterior cingulate gyri. Smaller baseline volumes of brain structures reflect increased neurodegeneration predicting disability. The development of DGM atrophy may drive disability accumulation irrespective of clinical phenotypes, thereby becoming a useful outcome measure in neuroprotective clinical trials [8].

GM atrophy progresses in a manner which follows brain regions that are both functionally and/or structurally related to each other. Taken together, these results suggest that already atrophic structures may be at risk for an even greater rate of tissue loss in patients who progress in terms of clinical disability [1] (Figure 1).

\section{Mechanism}

Redox, inflammatory/autoimmune, vascular, and neurodegenerative are some of the several components involved in the mechanisms in the pathophysiology of MS, understanding the redox processes, Knowing the schedule of initial changes in pathogenic factors and the key turning points involved in MS pathogenesis, is the way to enable early treatment, and the development of therapies that target specific pathophysiological components of MS, which could alleviate the symptoms and hopefully stop MS.

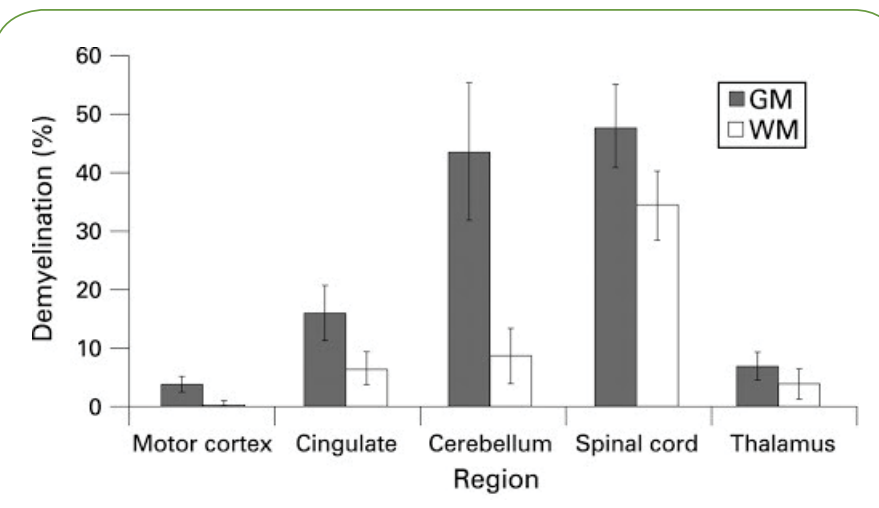

Figure 1 Graph explaining the Demyelination.

\section{References}

1 Bergsland N, Horakova D, Dwyer MG, Uher T, Vaneckova M, et al. (2018) Gray matter atrophy patterns in multiple sclerosis: A 10-year source-based morphometry study. Neuroimage Clin 17: 444-451.

2 De Stefano N, Giorgio A, Battaglini M, Rovaris M, Sormani MP, et al. (2010) Assessing brain atrophy rates in a large population of untreated multiple sclerosis subtypes. Neurology 74: 1868-1876.

3 Chataway J, Schuerer N, Alsanousi A, Chan D, MacManus D, et al. (2014) Effect of high-dose simvastatin on brain atrophy and disability in secondary progressive multiple sclerosis (MS-STAT): A
The negative symptoms during relapses are paralysis, blindness and numbness. The main reason behind those is the conduction block caused largely by demyelination and inflammation, and possibly by defects in synaptic transmission and putative circulating blocking factors. Recoveries from symptoms happen mainly due to the restoration of axonal properties. Axonal restoration happens either by remyelination, or the resolution of inflammation. An important mechanism determining persistent neurological deficit is axonal degeneration in deep grey matter.

\section{Treatment}

When talking about MS or the first MS event the starting treatment which should be done abruptly is to treats all symptoms and slows the progression of the disease internally by decreasing the frequency of relapses.

There are diverse MS treatment options available today. The treatments are given subcutaneous, intramuscular, intravenous injection or orally [9]. Beta interferons and Glatiramer acetate are the used for the treatment of relapsing-remitting MS via injections [10]. Immunomodulatory therapy is used for the underlying immune disorder and management of symptoms. Even non-pharmacologic treatments, such as physical and occupational therapy are also advisable.

\section{Aetiology}

Different hypotheses are there based on the causes of MS. A convoluted web of gender, ethnicity, and heredity, with infectious diseases and environmental factors are also playing roles. Similarly, vitamin D deficiency and smoking also been attributed to increased susceptibility to MS.

\section{Conclusion}

In a nut shell, MS is a severe neurodegenerative disease that has numerous effects on the human body's functional ability. And GM atrophy occurs as a regional rather than global process in MS, and that functional disability is specifically associated with atrophy of the left pre/post central gyrus. In the research discussed it is evident that the increased disability in MS is followed by the decrease in grey matter over time. The early detection and the precise medications for this malaise can decrease the adverse manifestations.

4 https://clinicaltrials.gov/ct2/show/study/NCT01910259?term $=$ mssmart\&rank $=1$

5 Geurts JJG, Calabrese M, Fisher E, Rudick RA (2012) Measurement and clinical effect of grey matter pathology in multiple sclerosis. Lancet Neurol 11: 1082-1092.

6 Steenwijk MD, Geurts JJG, Daams M, Tijms BM, Wink AM, et al. (2016) Cortical atrophy patterns in multiple sclerosis are nonrandom and clinically relevant. Brain 139: 115-126.

7 Eshaghi A, Bodini B, Ridgway GR, García-Lorenzo D, Tozer DJ, et al. 
(2014) Temporal and spatial evolution of grey matter atrophy in primary progressive multiple sclerosis. Neurolmage 86: 257-264.

8 Eshaghi A, Prados F, Brownlee W, Altmann DR, Tur C, et al. (2018) Deep grey matter volume loss drives disability worsening in multiple sclerosis. Ann Neurol (in press).
9 Hooper K (2011) Managing progressive MS. New York, NY: National Multiple Sclerosis Society.

10 Hooper K (2011) Multiple sclerosis: just the facts. New York, NY; National Multiple Sclerosis Society. 